\title{
Treatment of pretibial myxedema with intralesional immunomodulating therapy
}

This article was published in the following Dove Press journal:

Therapeutics and Clinical Risk Management

8 September 2017

Number of times this article has been viewed

\section{Ziwei Ren ${ }^{1, *}$ \\ Min $\mathrm{He}^{2, *}$ \\ Fang Deng ${ }^{2}$ \\ Yan Chen' \\ Liyin Chai' \\ Bing Chen ${ }^{2}$ \\ Wuquan Deng' \\ 'Department of Endocrinology and Nephrology, Chongqing Emergency Medical Hospital (The Fourth People's Hospital of Chongqing), ${ }^{2}$ Department of Endocrinology, Southwest Hospital, Third Military Medical University, Chongqing, People's Republic of China \\ *These authors contributed equally to this work}

Correspondence: Wuquan Deng Department of Endocrinology and Nephrology, Chongqing Emergency Medical Hospital (The Fourth People's Hospital of Chongqing), Nol Jiankang Road, Yuzhong District, Chongqing, People's Republic of China Tel +862363692185

Email wuquandeng@gmail.com
Objective: Local immune regulation therapy has been one of the therapeutic methods used for the treatment of autoimmune thyroid disease in patients with pretibial myxedema (PTM). However, the poor response rate and high recurrence rate are still major problems. Whether a premixed corticosteroid, compound betamethasone, could enhance remission rate and decrease recurrence rate in patients with PTM was investigated in the present study.

Subjects and methods: We have performed a clinical utility observation of compound betamethasone with intralesional injections based on basic thyroid disease treatment in 32 PTM patients between January 2008 and August 2016. The patients were followed up for 2 years, and the clinical outcomes and side effects were calculated and analyzed.

Results: All patients had a complete remission after different times of injection. A total of $21.7 \%$ patients had complete remission with one time of injection, $34.8 \%$ with two times of injection, $17.4 \%$ with three times of injection, $4.3 \%$ with four times of injection, and $4.3 \%$ with five times of injection. In all, $56.3 \%$ patients with a disease duration of $<6$ months had complete remission after a 1-month treatment, 37.5\% patients with a disease duration between 6 months and 12 months had complete remission after a 2-month treatment, 3.1\% patients with a disease duration of 2 years had complete remission after a 5 -month treatment, and $3.1 \%$ with a disease duration of 5 years had complete remission after a 7-month treatment.

Conclusion: Compound betamethasone with multipoint intralesional injection is a feasible, effective, and secure novel strategy in the treatment of PTM.

Keywords: pretibial myxedema, glucocorticoids, compound betamethasone, intralesional injection

\section{Introduction}

Thyroid disease and its complications have been recognized as one of the most important economic burden and social issues all over the world, especially in developing countries. Pretibial myxedema (PTM; also called thyroid dermopathy, localized myxedema, or infiltrative dermopathy) is an infrequent manifestation of autoimmune thyroid disease. It is common in Graves' disease (GD) with an incidence of $0.5 \%-4.3 \%,{ }^{1}$ a cause of hyperthyroidism. GD is characterized by three main specifically extrathyroidal manifestations: PTM, Graves' ophthalmopathy (GO), and thyroid acropachy. ${ }^{2,3}$ PTM also occurs in patients with primary hypothyroidism and Hashimoto's thyroiditis (HT). Interestingly, PTM has been reported, but very rarely, in patients with no past or present thyroid dysfunction. Featured with circumscribed localized myxedema and thickening of the skin, ${ }^{4,5}$ PTM is a form of diffuse mucinosis in which there is an accumulation of excess glycosaminoglycans in the dermis and subcutis of the skin. Glycosaminoglycans, also called mucopolysaccharides, are complex carbohydrates that are important for tissue hydration and lubrication. The main glycosaminoglycan in pretibial myxedema is 
hyaluronic acid, which is made by cells called the fibroblasts. The most common present lesions of PTM are non-pitting edema and plaque forms. Nodular pretibial dermopathy and elephantiasis forms usually occur in serious cases. ${ }^{6,7}$

The common clinical symptom and sign of PTM is invasive skin lesion on the shins (pretibial areas), acrotarsium, and toe. PTM has also been reported, but much less than, to occur on the upper extremities, particularly in areas exposed to repeated trauma, surgical scars, vaccination sites, and burn scars. ${ }^{8-10}$ In addition, buttock nodules and ureteral myxedema have been reported in patients with GD. ${ }^{11,12}$ PTM patients with mild condition are usually asymptomatic, but severe cases may induce functional problems such as difficulty in dressing and wearing socks and shoes. Only few patients could have painful, pruritic, and hyperpigmentation conditions. ${ }^{6,13}$ In some cases, entrapment neuropathy and even foot drop have been reported. ${ }^{14}$ It is amazing that PTM could masquerade as a venous leg ulcer so that PTM is under-recognized because both of them have a similar clinical presentation. ${ }^{15}$ Meanwhile, in the elder patients with severe conditions and concomitant disease, it may influence body balance or even ambulatory function. ${ }^{16}$ Quality of life of involved patients is obviously affected, and therapeutic approach is necessary and urgent. ${ }^{4,5,13,17,18}$

A large number of therapeutic approaches have been proposed to prevent processes of PTM and relieve the clinical symptoms. Compression stockings and intermittent pump have been used for patients with lymphedema. ${ }^{6,19}$ Complete decompressive physiotherapy and graduated compressive bandaging could also be beneficial in severe cases such as elephantiasis forms. ${ }^{6,20}$ Surgical excision was not recommended because of the possibility of surgical trauma-related aggravation. ${ }^{6}$ Plasmapheresis and cytotoxic therapy have been tried, but the efficacy of these therapies in PTM is not clear. Systemic corticosteroids utility should be avoided because of undesirable adverse effects, except in GO. Intralesional or topical corticosteroid therapy is currently the main therapeutic method in the treatment of PTM. ${ }^{21,22}$ Early administration of corticosteroid could prevent secondary processes such as fibrosis and lymphatic obstruction..$^{23,24}$ External application of fluocinolone acetonide, clobetasol propionate, and triamcinolone cream could be helpful. ${ }^{3,6}$ Intralesional multiple injections of a solution of dexamethasone, lidocaine, and saline are also reported to result in significant resolutions. ${ }^{19,25}$ Although local corticosteroid therapy has a certain curative effect on treatment of PTM, the poor response rate, high recurrence rate, and its adverse reactions are still huge problems. ${ }^{19,24}$ Therefore, the key to treatment of PTM was how to choose an appropriate glucocorticoid to increase the therapeutic effect and prevent recurrence. In the present study, compound betamethasone with intralesional injection was applied in PTM patients to certify the remission rate and recurrence rate.

\section{Subjects and methods Patients}

A total of 32 patients with PTM (12 males and 20 females) were randomly enrolled in the study. The average age of patients was 50 years (range 18-70 years). GD was diagnosed in 20 out of 32 patients with a mean duration of 3.5 years, and the mean duration of PTM was 2.0 years; five patients had posttreatment of GD with radioactive iodine, four had HT, two had primary hypothyroidism, and two had euthyroidism. There were 18 cases with diffuse PTM, 9 with nodular- or plaque-like myxedema, and 13 with elephantiasis. In all, 30 of 32 patients were diagnosed with GO. In 25 patients, GO was confirmed by skin biopsy. This study was performed from January 2008 through August 2016 and approved by the institutional review board of Southwest Hospital. All subjects provided written informed consent.

\section{Methods}

\section{Basic treatment}

Based on thyroid function, patients with hyperthyroidism were administered with antithyroid agents $(n=20)$, those with hypothyroidism with oral levothyroxine (LT4) replacement $(n=8)$, and those with euthyroidism without therapy $(n=1)$. Patients with fungal infection were treated with antifungal agents for external application.

\section{Topical therapy}

The skin lesions were disinfected regularly. In all, $1 \mathrm{~mL}$ of compound betamethasone (betamethasone sodium phosphate $2 \mathrm{mg}$ and betamethasone dipropionate $5 \mathrm{mg}$, Diprospan, Schering-Plough) was mixed sufficiently in a 1:5 dilution with $5 \mathrm{~mL}$ of $5 \%$ lidocaine in a $10 \mathrm{~mL}$ syringe. Multipoint intralesional injection was conducted with $0.1-0.5 \mathrm{~mL}$ of premixed solution in the skin lesions with a needle $0.5 \mathrm{~mm}$ in diameter. Compression hemostasis with aseptic cotton buds was applied after injection treatment. All skin lesions were performed with the same method with repeated intervals of $1 \mathrm{~cm}$. The solution was injected in the middle of nodules for the nodular myxedema, and fan-shaped injection was performed in large nodules. The treated area was covered with a sterile pad for 2 or 3 days after injection to avoid infection (Figures 1 and 2).

\section{Efficacy evaluation and follow-up}

According to the changes of PTM after treatment, we define efficacy based on following three change points: 1) complete remission: the edema subsided completely; 2) effective: nodule 

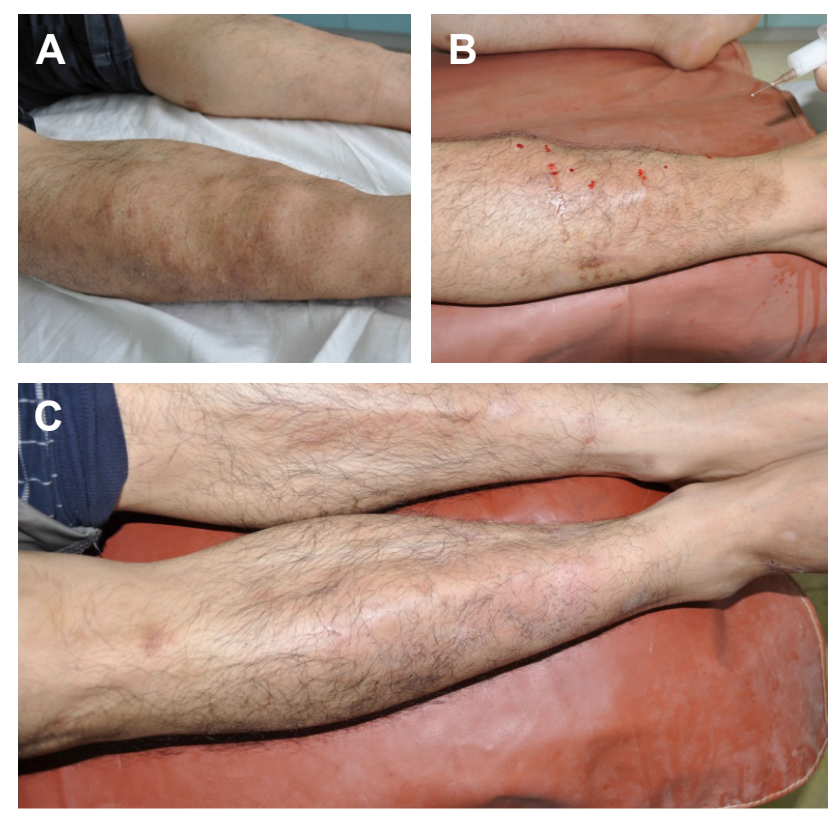

Figure I Nodular PTM treatment with compound betamethasone.

Notes: (A) Nodular PTM before treatment, (B) intralesional with compound betamethasone, (C) nodular PTM complete remission.

Abbreviation: PTM, pretibial myxedema.

decreased or partial remission; and 3) inefficient: the area of edema was not decreased or even deteriorated. A semimonthly return visit happened at the first 3 months and monthly then. The changes in skin lesions were observed when patients attended clinic, and a repetitive dose was given to those who had incomplete remission each time. The follow-up ended until PTM was completely subsided or after a treatment period of 2 years.

\section{Results}

All 32 cases had complete remission after different frequencies of drug administration: 7 (21.7\%) cases experienced complete remission and 17 (53.1\%) cases had effectiveness after first administration. After twice administration, 11 (34.8\%) cases had complete remission and 7 had effectiveness. There were six (17.4\%) cases of complete remittance, and two cases had effectiveness after three times of administration. In addition, six (17.4\%) cases had complete remission after four times, one (4.3\%) after eight times, and one (4.3\%) after ten times (Table 1). Considering the duration of disease, 18 cases with $<6$ months experienced complete remission after a 1-month treatment. In 12 cases whose disease duration was between 6 and 12 months, complete remission was attained after a 2-month treatment. One case with PTM for 2 years had a complete remission after a 5-month treatment, and one case with 5 years was treated for 7 months (Table 2).

The most common side effects were pain and bleeding at the injection site in the treatment process of PTM.
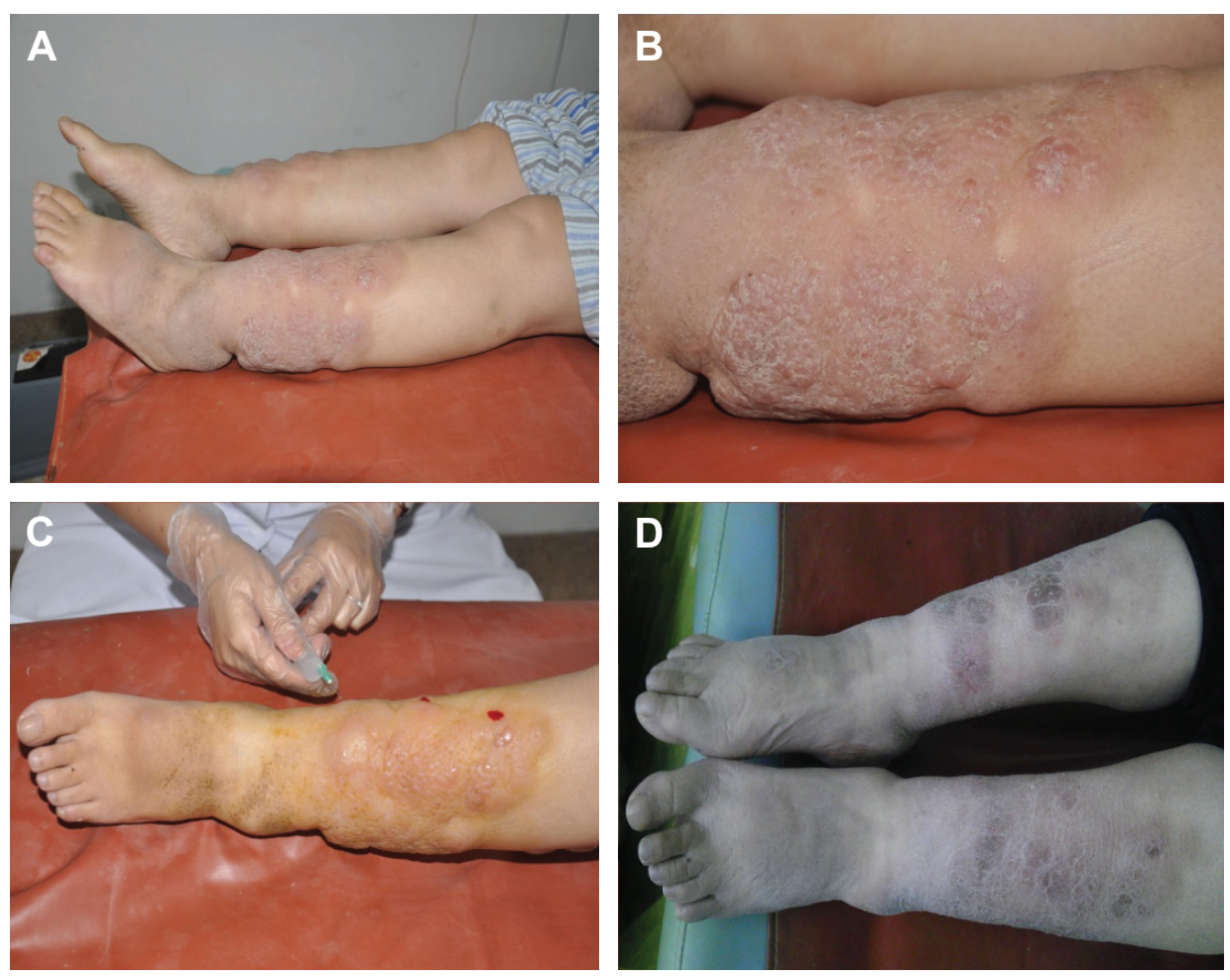

Figure 2 Elephantiasic PTM treatment with compound betamethasone.

Notes: (A and B) Elephantiasic PTM before treatment, (C) intralesional with compound betamethasone, (D) elephantiasic PTM complete remission. Abbreviation: PTM, pretibial myxedema. 
Table I Recovery of patients after treatment according to the administered frequency

\begin{tabular}{|c|c|c|c|c|c|c|}
\hline $\begin{array}{l}\text { Administered } \\
\text { frequency, } \\
\text { number of times }\end{array}$ & I & 2 & 3 & 4 & 8 & 10 \\
\hline $\begin{array}{l}\text { Complete } \\
\text { remission, n (\%) }\end{array}$ & $7(2 \mid .7)$ & II (34.8) & $6(17.4)$ & $6(17.4)$ & I (4.3) & I (4.3) \\
\hline Effective, n (\%) & $17(53.1)$ & $7(21.7)$ & $2(6.25)$ & $2(6.25)$ & I (3.13) & - \\
\hline
\end{tabular}

Note: '-' indicates no data available at ten times administered frequency.

These symptoms could relieve with compressing hemostasis for a few minutes and with no need for additional treatment. No patient had local infection, ecchymosis, and skin atrophy in this study. In all, 32 patients had an average follow-up time of 18 months and 2 patients were lost. A total of 25 patients had no recurrence in original skin lesions and no systemic adverse reaction occurred. One patient with diffuse PTM had local skin lesions relapse, while the skin lesions disappeared after treated twice again. Two patients with elephantiasis type of PTM had relapsed. Their skin lesions disappeared after two and three times of injection separately.

\section{Discussion}

As one of the specific clinical manifestation of its complication in autoimmune thyroid disease, lower extremity myxedema usually accompanies with GD, HT, and so on. Most of the patients may have mild symptoms, but a severe condition can seriously affect the quality of life. Some patients had pruritus and hyperpigmentation. Huge and wide spread myxedema nodule could influence on foot wearing and outlook. It also could cause a bad confidence and even a serious mental illness. Therefore, a minor harmful therapy would be vital for relieving it. Although local immune regulation therapy has been reported as an important and effective therapeutic method in myxedema nodule, poor response and high relapse rate were still huge problems. Therefore, the key to treatment of myxedema nodule was how to choose glucocorticoid properly to increase treatment effect and prevent recurrence. Previous studies have revealed that longacting glucocorticoid, dexamethasone, and triamcinolone acetonide had an ideal effect on relieving of myxedema

Table 2 Recovery of patients after treatment according to the duration of disease

\begin{tabular}{lllll}
\hline Duration of disease & $<\mathbf{6}$ months & $\mathbf{6 - 1 2}$ months & $\mathbf{2}$ years & $\mathbf{5}$ years \\
\hline $\begin{array}{l}\text { Number of } \\
\text { patients, } \mathrm{n}(\%)\end{array}$ & $18(56.3)$ & $12(37.5)$ & $\mathrm{I}(3.1)$ & $\mathrm{I}(3.1)$ \\
$\begin{array}{l}\text { Recovery time, } \\
\text { months }\end{array}$ & $\mathrm{I}$ & 2 & 5 & 7 \\
\hline
\end{tabular}

nodule but had a disappointing recurrence problem. The approach of treatment and drugs would directly influence the efficacy of the disease. ${ }^{26-28}$ According to pathogenesis of disease, we chose a short-acting and long-acting premixed glucocorticoid solution in clinical practice and achieved a good clinical utility. Betamethasone dipropionate and betamethasone sodium phosphate could have a "double effect" for PTM. Betamethasone dipropionate could take effect in a short period of time after intralesional injection, while betamethasone sodium phosphate was slowly absorbed with longer effective time.

The etiology of PTM remains unclear so far. It is generally considered as an autoimmune reaction dominantly caused by autoantibodies. ${ }^{6,29} \mathrm{~A}$ high concentration of thyroidstimulating hormone (TSH) receptor antibodies was found in patients with PTM. The thyrotrophin receptor located in skin lesion may be attacked by the TSH receptor antibody in the body and trigger an autoimmune damage. A large number of glycosaminoglycans were produced by fibroblast when it was stimulated by the activation of humoral immunity and cellular immunity response. The regional myxedema was developed because of glycosaminoglycans deposition in the dermis and subcutis. The immunosuppressive effect of long-acting glucocorticoid that it had a certain curative effect to treat PTM had been reported, such as local injection of triamcinolone acetonide or blockage. ${ }^{24,25,30}$ However, the defect of monocomponent caused the effect to be slow and unstable, as well as easy to relapse. ${ }^{30,31}$ Therefore, the compound betamethasone, a premixed glucocorticoid of betamethasone phosphate disodium and betamethasone dipropionate, had been used for PTM in this study. The betamethasone phosphate disodium could be absorbed rapidly for its good solubility properties, and the plasma concentration would reach a peak at 1 hour. At the same time, betamethasone dipropionate was slightly dissolved and slowly absorbed. It took effect for a long continuous time at the injection site. Based on our clinical practice, it was demonstrated that compound betamethasone with intralesional therapy had a rapidly effective, safe, and long-time acting response for PTM. Moreover, it had almost no side effects and lesions. The probable mechanisms of compound betamethasone are based on two points. The first one is local immunoregulatory therapy: by creating a partial high concentration of glucocorticoid, the mixture stables the membrane's structure, controls inflammation, and reduces the immune response with direct contact and slow infiltration. It also reduces the production of hyaluronic acid and granules in mastocytes. Thus, the deposition of mucopolysaccharides and glycosaminaglycans was decreased, and the 
infiltration of phagocytes, plasma cells, and fibroblasts was all inhibited. The second one is compound betamethasone with a short-acting and long-acting premixed glucocorticoid solution that has a double effect. Hence, it could rapidly take and maintain its curative effect for PTM. Meanwhile, we found that clinical efficacy was related to disease duration and range of skin lesions. In this study, one patient with a disease duration of 5 years and diffuse type of PTM and two patients with elephantiasis type of PTM were relapsed. It indicated that PTM patients with a shorter disease duration and smaller skin lesions had higher effective rate and lower relapse rate. Therefore, early diagnosis and treatment is very important to patient's prognosis.

There are some points for attention: First, the solution should be sufficiently blended before injection. Second, in order to avoid dermal atrophy and fragility, the total solution should not be excessive and interval distance should be enough for each injection. Third, the appropriate depths of needle inserted should be considered based on lesion size. The absorption of liquid medicine would be influenced by whether the needle was too deep or too shallow. Finally, as we observed, skin atrophy and hyperpigmentation could occur in treated areas with the atrophy of subcutaneous adipose tissue. However, dimpling of local lesions usually was caused by skin biopsy, not by topical injection.

Local immunoregulatory therapy of compound betamethasone with intralesional injection was a practical, feasible, and safe treatment method for patients with PTM. However, it should also be paid attention whether it is necessary with a combination therapy, because PTM is a systemic autoimmune disease. Meanwhile, some study limitations must be considered. First, the present study is an observational study. Therefore, a randomized controlled trial is necessary to further verify clinical utility in the future. Second, it is a case series study; a large number of subjects should be enrolled in future studies. A few of novel approaches have been reported previously, including intravenous high-dose corticosteroid therapy, immunosuppressive agents (such as cyclophosphamide), immunomodulators (such as immunoglobulin and octreotide), ${ }^{32}$ or novel medications (rituximab and plasmapheresis). ${ }^{33}$ Therefore, it remains to be certified the clinical utility of compound betamethasone with further clinical and basic experimental studies in the future.

\section{Acknowledgment}

The project supported by Chongqing medical program for cultivation of high-end talent reserves for Wuquan Deng.

\section{Disclosure}

The authors report no conflicts of interest in this work.

\section{References}

1. Kriss JP. Pathogenesis and treatment of pretibial myxedema. Endocrinol Metab Clin North Am. 1987;16(2):409-415.

2. Bartalena L, Tanda ML. Clinical practice. Graves' ophthalmopathy. N Engl J Med. 2009;360(10):994-1001.

3. Fatourechi V. Thyroid dermopathy and acropachy. Best Pract Res Clin Endocrinol Metab. 2012;26(4):553-565.

4. Cannavo SP, Borgia F, Vaccaro M, Guarneri F, Magliolo E, Guarneri B. Pretibial myxoedema associated with Hashimoto's thyroiditis. J Eur Acad Dermatol Venereol. 2002;16(6):625-627.

5. Georgala S, Katoulis AC, Georgala C, Katoulis EC, Hatziolou E, Stavrianeas NG. Pretibial myxedema as the initial manifestation of Graves' disease. J Eur Acad Dermatol Venereol. 2002;16(4):380-383.

6. Bartalena L, Fatourechi V. Extrathyroidal manifestations of Graves' disease: a 2014 update. J Endocrinol Invest. 2014;37(8):691-700.

7. Fatourechi V. Pretibial myxedema: pathophysiology and treatment options. Am J Clin Dermatol. 2005;6(5):295-309.

8. Rice SA, Peden NR, McGlynn S, Morton C. Atypical presentation of infiltrative thyroid dermopathy. Clin Exp Dermatol. 2010;35(1):56-58.

9. Noppakun N, Bancheun K, Chandraprasert S. Unusual locations of localized myxedema in Graves' disease. Report of three cases. Arch Dermatol. 1986;122(1):85-88.

10. Dhaille F, Dadban A, Meziane L, et al. Elephantiasic pretibial myxoedema with upper-limb involvement, treated with low-dose intravenous immunoglobulins. Clin Exp Dermatol. 2012;37(3):307-308.

11. Cao Y, Zhu HJ, Zheng HY, Li J. Myxedema. Lancet Diabetes Endocrinol. 2014;2(7):600.

12. Minutoli F, Benedetto C, Visalli C, Granata A, Gaeta M. Computed tomography and magnetic resonance imaging findings of ureteral myxedema in Graves' disease. Clin Imaging. 2015;39(4):711-713.

13. Lohiya S, Lohiya V, Stahl EJ. Pretibial myxedema without ophthalmopathy: an initial presentation of Graves' disease. Am J Med Sci.2013;346(1): 73-75.

14. Siegler M, Refetoff S. Pretibial myxedema-a reversible cause of foot drop due to entrapment of the peroneal nerve. N Engl J Med. 1976;294(25): $1383-1384$.

15. Herskovitz I, Hughes O, MacQuhae F, Kirsner RS. Pretibial myxedema masquerading as a venous leg ulcer. Wounds. 2017;29(3):77-79.

16. Zhou H, Sabbagh M, Wyman R, Liebsack C, Kunik ME, Najafi B. Instrumented trail-making task to differentiate persons with no cognitive impairment, amnestic mild cognitive impairment, and Alzheimer disease: a proof of concept study. Gerontology. 2017;63(2):189-200.

17. Bilen H, Atasoy M, Akcay G, et al. Elephantiasic pretibial myxedema and cutis verticis gyrata caused by Graves' disease. Thyroid. 2006;16(8): 815-816.

18. Lu Y-Y, Wei K-C. Elephantiasic pretibial myxedema. Intern Med. 2012; 51(19):2837-2837.

19. Vannucchi G, Campi I, Covelli D, Forzenigo L, Beck-Peccoz P, Salvi M. Treatment of pretibial myxedema with dexamethasone injected subcutaneously by mesotherapy needles. Thyroid. 2013;23(5):626-632.

20. Susser WS, Heermans AG, Chapman MS, Baughman RD. Elephantiasic pretibial myxedema: a novel treatment for an uncommon disorder. J Am Acad Dermatol. 2002;46(5):723-726.

21. Lan C, Li C, Chen W, Mei X, Zhao J, Hu J. A randomized controlled trial of intralesional glucocorticoid for treating pretibial myxedema. J Clin Med Res. 2015;7(11):862-872.

22. Ramos LO, Mattos PC, Figueredo GL, Maia AA, Romero SA. Pre-tibial myxedema: treatment with intralesional corticosteroid. An Bras Dermatol. 2015;90(3 Suppl 1):143-146.

23. Schwartz KM, Fatourechi V, Ahmed DD, Pond GR. Dermopathy of Graves' disease (pretibial myxedema): long-term outcome. J Clin Endocrinol Metab. 2002;87(2):438-446. 
24. Takasu N, Higa H, Kinjou Y. Treatment of pretibial myxedema (PTM) with topical steroid ointment application with sealing cover (Steroid Occlusive Dressing Technique: Steroid ODT) in Graves' Patients. Intern Med. 2010;49(7):665-669.

25. Deng A, Song D. Multipoint subcutaneous injection of long-acting glucocorticoid as a cure for pretibial myxedema. Thyroid. 2011; 21(1):83-85.

26. Jolles S, Hughes J. Use of IGIV in the treatment of atopic dermatitis, urticaria, scleromyxedema, pyoderma gangrenosum, psoriasis, and pretibial myxedema. Int Immunopharmacol. 2006;6(4):579-591.

27. Shih SR, Lin MS, Li HY, et al. Observing pretibial myxedema in patients with Graves' disease using digital infrared thermal imaging and high-resolution ultrasonography: for better records, early detection, and further investigation. Eur J Endocrinol. 2011;164(4): 605-611.

28. Yu H, Jiang X, Pan M, Huang R. Elephantiasic pretibial myxedema in a patient with graves disease that resolved after 131I treatment. Clin Nucl Med. 2014;39(8):758-759.
29. Kim WB, Mistry N, Alavi A, Sibbald C, Sibbald RG. Pretibial myxedema: case presentation and review of treatment options. Int J Low Extrem Wounds. 2014;13(2):152-154.

30. Kamath C, Young S, Kabelis K, et al. Thyrotrophin receptor antibody characteristics in a woman with long-standing Hashimoto's who developed Graves' disease and pretibial myxoedema. Clin Endocrinol (Oxf). 2012;77(3):465-470.

31. Hadj-Kacem H, Rebuffat S, Mnif-Feki M, Belguith-Maalej S, Ayadi H, Peraldi-Roux S. Autoimmune thyroid diseases: genetic susceptibility of thyroid-specific genes and thyroid autoantigens contributions. Int $J$ Immunogenet. 2009;36(2):85-96.

32. Shinohara M, Hamasaki Y, Katayama I. Refractory pretibial myxoedema with response to intralesional insulin-like growth factor 1 antagonist (octreotide): downregulation of hyaluronic acid production by the lesional fibroblasts. Br J Dermatol. 2000;143(5):1083-1086.

33. Heyes C, Nolan R, Leahy M, Gebauer K. Treatment-resistant elephantiasic thyroid dermopathy responding to rituximab and plasmapheresis. Australas J Dermatol. 2012;53(1):e1-e4.
Therapeutics and Clinical Risk Management

\section{Publish your work in this journal}

Therapeutics and Clinical Risk Management is an international, peerreviewed journal of clinical therapeutics and risk management, focusing on concise rapid reporting of clinical studies in all therapeutic areas outcomes, safety, and programs for the effective, safe, and sustained use of medicines. This journal is indexed on PubMed Central, CAS,

\section{Dovepress}

EMBase, Scopus and the Elsevier Bibliographic databases. The manuscript management system is completely online and includes a very quick and fair peer-review system, which is all easy to use. Visit http://www.dovepress.com/testimonials.php to read real quotes from published authors. 